\title{
Article \\ CRISPRi-Guided Metabolic Flux Engineering for Enhanced Protopanaxadiol Production in Saccharomyces cerevisiae
}

\author{
Soo-Hwan Lim ${ }^{1, \dagger}{ }^{\text {, Jong-In Baek }}{ }^{2,+}{ }^{\circledR}$, Byeong-Min Jeon ${ }^{2} \mathbb{D}$, Jung-Woo Seo ${ }^{1}$, Min-Sung Kim ${ }^{2}$, Ji-Young Byun ${ }^{2}$, \\ Soo-Hoon Park ${ }^{1}$, Su-Jin Kim ${ }^{1}$, Ju-Young Lee ${ }^{3}$, Jun-Hyoung Lee ${ }^{1}$ and Sun-Chang Kim ${ }^{1,2,4, *}$ \\ 1 Intelligent Synthetic Biology Center, 291 Daehak-ro, Daejeon 305-701, Korea; lshbio@kaist.ac.kr (S.-H.L.); \\ jwseo123@kaist.ac.kr (J.-W.S.); shpark11@guest.kaist.ac.kr (S.-H.P.); food7568@gmail.com (S.-J.K.); \\ junhlee@kaist.ac.kr (J.-H.L.) \\ 2 Department of Biological Sciences, Korea Advanced Institute of Science and Technology, 291 Daehak-ro, \\ Daejeon 305-701, Korea; baekji@kaist.ac.kr (J.-I.B.); jbm0901@kaist.ac.kr (B.-M.J.); \\ mskim8906@kaist.ac.kr (M.-S.K.); byunjy@kaist.ac.kr (J.-Y.B.) \\ 3 Research Center for Bio-Based Chemistry, Korea Research Institute of Chemical Technology (KRICT), 406-30, \\ Jongga-ro, Ulsan 44429, Korea; juylee@krict.re.kr \\ 4 KAIST Institute for Biocentury, Korea Advanced Institute of Science and Technology, 291 Daehak-ro, \\ Daejeon 305-701, Korea \\ * Correspondence: sunkim@kaist.ac.kr; Tel.: +82-42-350-2619 \\ + These authors contributed equally to this work.
}

check for updates

Citation: Lim, S.-H.; Baek, J.-I.; Jeon, B.-M.; Seo, J.-W.; Kim, M.-S.; Byun, J.-Y.; Park, S.-H.; Kim, S.-J.; Lee, J.-Y.; Lee, J.-H.; et al. CRISPRi-Guided Metabolic Flux Engineering for Enhanced Protopanaxadiol Production in Saccharomyces cerevisiae. Int. J. Mol. Sci. 2021, 22, 11836. https://doi.org/ $10.3390 /$ ijms222111836

Academic Editor: Joaquin Arino

Received: 24 September 2021

Accepted: 28 October 2021

Published: 31 October 2021

Publisher's Note: MDPI stays neutral with regard to jurisdictional claims in published maps and institutional affiliations.

Copyright: (c) 2021 by the authors. Licensee MDPI, Basel, Switzerland. This article is an open access article distributed under the terms and conditions of the Creative Commons Attribution (CC BY) license (https:/ / creativecommons.org/licenses/by/ $4.0 /)$.

\begin{abstract}
Protopanaxadiol (PPD), an aglycon found in several dammarene-type ginsenosides, has high potency as a pharmaceutical. Nevertheless, application of these ginsenosides has been limited because of the high production cost due to the rare content of PPD in Panax ginseng and a long cultivation time (4-6 years). For the biological mass production of the PPD, de novo biosynthetic pathways for PPD were introduced in Saccharomyces cerevisiae and the metabolic flux toward the target molecule was restructured to avoid competition for carbon sources between native metabolic pathways and de novo biosynthetic pathways producing PPD in S. cerevisiae. Here, we report a CRISPRi (clustered regularly interspaced short palindromic repeats interference)-based customized metabolic flux system which downregulates the lanosterol (a competing metabolite of dammarenediol-II (DD-II)) synthase in S. cerevisiae. With the CRISPRi-mediated suppression of lanosterol synthase and diversion of lanosterol to DD-II and PPD in S. cerevisiae, we increased PPD production 14.4-fold in shake-flask fermentation and 5.7-fold in a long-term batch-fed fermentation.
\end{abstract}

Keywords: CRISPR interference; lanosterol; protopanaxadiol; triterpenes; metabolic engineering; ginsenosides; Saccharomyces cerevisiae

\section{Introduction}

Protopanaxadiol (PPD), an aglycon of dammarene-type ginsenosides, possesses anticancer, anti-inflammatory, anti-oxidant, hepatoprotectant, anti-lipogenic, wound-healing, and anti-obesity activity [1-5]. Despite having high pharmacological potency, the application of these ginsenosides as drugs and pharmaceuticals is limited because Panax ginseng cultivation is slow, and the ginsenoside content of ginseng is low [6]. To overcome this, PPD and ginsenosides have been produced using de novo biosynthetic pathways in heterologous microbial organisms, including yeast [7-12]. PPD is biosynthesized from 2,3-oxidosqualene, which is derived from isopentenyl diphosphate and dimethylallyl diphosphate ([13]. These precursors are synthesized via the mevalonate (MVA) pathway in both ginseng and yeast [14]. PPD biosynthesis from 2,3-oxidosqualene in yeast requires three ginseng-derived enzymes-P. ginseng dammarenediol-II (DD-II) synthase (PgDS), P. ginseng PPD synthase (PgPPDS), and P. ginseng cytochrome P450 reductase (PgCPR1) [15-17]. PgDS, an oxidosqualene cyclase, converts 2,3-oxidosqualene to DD-II. PgPPDS and PgCPR1 together catalyze the hydroxylation of DD-II at the C-12 position, 
resulting in PPD biosynthesis. PPD then undergoes glycosylation catalyzed by several UDP-glucosyltransferases, yielding ginsenosides [13].

In yeast, however, 2,3-oxidosqualene is natively converted to lanosterol, catalyzed by lanosterol synthase, and then used for biosynthesis of sterols such as ergosterol [18]. Thus, in yeast, the PPD biosynthetic pathway competes with the native sterol biosynthetic pathway for 2,3-oxidosqualene. Since deletion of the ERG7 gene, which encodes lanosterol synthase in yeast, is lethal, it is not possible to completely block the sterol biosynthetic pathway [18]. Rather, it is necessary to quantitatively control ERG7 expression (i.e., partial suppression) in order to enhance the PPD biosynthetic pathway. For example, in Saccharomyces cerevisiae, mass production of heterologous terpenes, which are synthesized from MVA, requires partial suppression of the metabolic flux of native sterol biosynthesis, in order to increase the metabolic flux of the target terpenes; this is achieved via replacement of the native promoter with the methionine-repressible MET3 promoter, the copper-repressible CTR3 promoter, or a constitutively weak promoter, or via fusion of the target enzyme to a degradation tag [19-26]. However, this strategy has several limitations. First, adding methionine increases production costs because it is consumed by yeast. Second, adding copper can result in toxicity in yeast. Third, it is difficult to quantitatively control the metabolic flux by replacing the native promoter with a constitutively weak promoter; further, selection of a suitable promoters is labor-intensive and time-consuming [27-30].

To address this, we developed a CRISPRi-based PPD biosynthesis approach for yeast. We constructed a PPD-producing yeast strain and engineered it to respond to increasing levels of the precursors. This approach alters cellular carbon flux and partially suppresses competing metabolic pathways to improve PPD production in yeast.

\section{Results}

\subsection{Construction of the PPD-Producing Yeast Strain}

Because dammarenediol-II synthase (DS), protopanaxadiol synthase (PPDS), and cytochrome P450 reductase (CPR) are necessary to construct the PPD-producing yeast strain, we first constructed $P g D S$ and PgPPDS expression cassettes that are controlled by the GPD promoter and a $P g C P R$ expression cassette that is controlled by the PGK1 promoter (Figure 1); these three cassettes were then integrated into the CEN.PK2-1D strain, producing the PPD-A1 strain (Table 1).

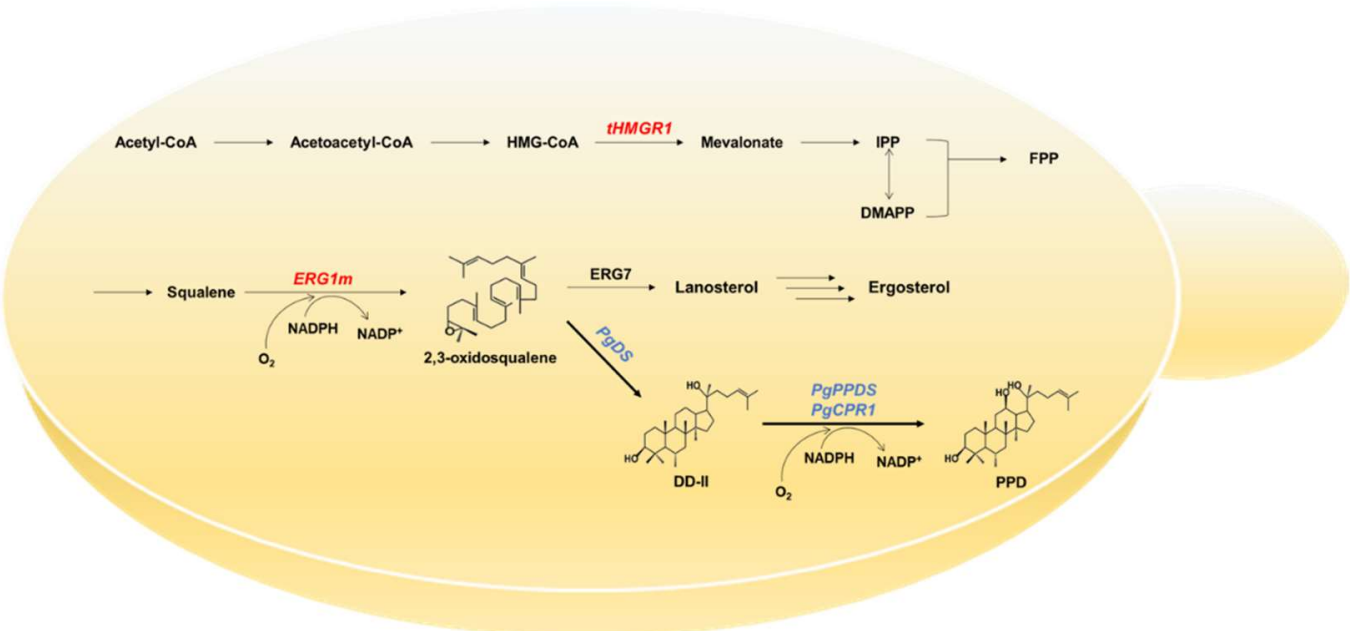

Figure 1. A biosynthetic pathway for protopanaxadiol (PPD) production in the metabolically engineered Saccharomyces cerevisiae. Blue: heterologous genes from Panax ginseng; red: engineered genes from S. cerevisiae. HMG-CoA, 3-hydroxyl3-methylglutaryl coenzyme A; IPP, isopentenyl pyrophosphate; DMAPP, dimethylallyl pyrophosphate; FPP, farnesyl diphosphate; DD-II, dammarenediol-II; PgDS, P. ginseng dammarenediol-II synthase; PgPPDS, P. ginseng protopanaxadiol synthase; and PgCPR, P. ginseng cytochrome P450 reductase. 
Table 1. Strains used in this study.

\begin{tabular}{|c|c|c|}
\hline & Description & Source \\
\hline CEN.PK2-1D & 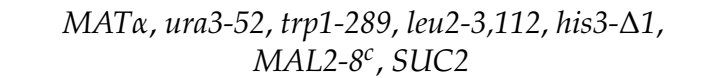 & EUROSCARF \\
\hline PPD-A1 & $\begin{array}{c}\text { GPDpro-DS-CYC1ter, GPDpro-PgPPDS-CYC1ter, } \\
\text { PGK1pro-PgCPR1-CYC1ter cassettes were inserted } \\
\text { into CEN.PK2-1D }\end{array}$ & This study \\
\hline PPD-A2 & $\begin{array}{c}\text { GPDpro-tHMGR1-CYC1ter cassette was inserted } \\
\text { into PPD-A1 }\end{array}$ & This study \\
\hline PPD-A3 & $\begin{array}{c}\text { TEF1pro-ERG1m-CYC1ter cassette was inserted } \\
\text { into PPD-A2 }\end{array}$ & This study \\
\hline PPD-A3-sgRNA1 & $\begin{array}{c}\text { [GPDpro-dCas9-ADH1ter]-[SNR52pro- } \\
\text { sgRNA1 } 1_{E R G 7 \text { pro-SUP4ter] cassette was inserted }} \\
\text { into PPD-A3 }\end{array}$ & This study \\
\hline PPD-A3-sgRNA2 & $\begin{array}{c}\text { [GPDpro- } d \text { Cas } 9-A D H 1 \text { ter]-[SNR52pro- } \\
\text { sgRNA2 ERG7pro-SUP4ter] cassette was inserted } \\
\text { into PPD-A3 }\end{array}$ & This study \\
\hline PPD-A3-sgRNA3 & $\begin{array}{c}\text { [GPDpro-dCas9-ADH1ter]-[SNR52pro- } \\
\text { sgRNA3 }{ }_{E R G 7 \text { pro-SUP4ter] cassette was inserted }} \\
\text { into PPD-A3 }\end{array}$ & This study \\
\hline PPD-A3-sgRNA4 & $\begin{array}{c}{[G P D \text { pro- } d \text { Cas } 9-A D H 1 \text { ter]-[SNR52pro- }} \\
\text { sgRNA4 } \\
\text { ERG7pro-SUP4ter] cassette was inserted } \\
\text { into PPD-A3 }\end{array}$ & This study \\
\hline PPD-A3-sgRNA5 & $\begin{array}{c}\text { [GPDpro-dCas9-ADH1ter]-[SNR52pro- } \\
\text { sgRNA5 } 5_{E R G 7 \text { pro-SUP4ter] cassette was inserted }} \\
\text { into PPD-A3 }\end{array}$ & This study \\
\hline
\end{tabular}

To evaluate PPD production in the PPD-A1 strain, we performed shake-flask fermentation using strains CEN.PK2-1D and PPD-A1 in YPD medium for $48 \mathrm{~h}$ (Figure 2). In the CEN.PK2-1D strain, squalene, 2,3-oxidosqualene, lanosterol, and ergosterol were produced at 21.3, 2.8, 9.9, and $49.7 \mathrm{mg} / \mathrm{L}$, respectively. In the PPD-A1 strain, PPD was produced at $1.5 \mathrm{mg} / \mathrm{L}$. DD-II was not accumulated, suggesting that it was completely converted to PPD. The amounts of lanosterol and ergosterol produced were similar in both strains. These results verify that the PPD-A1 strain produced PPD.

A

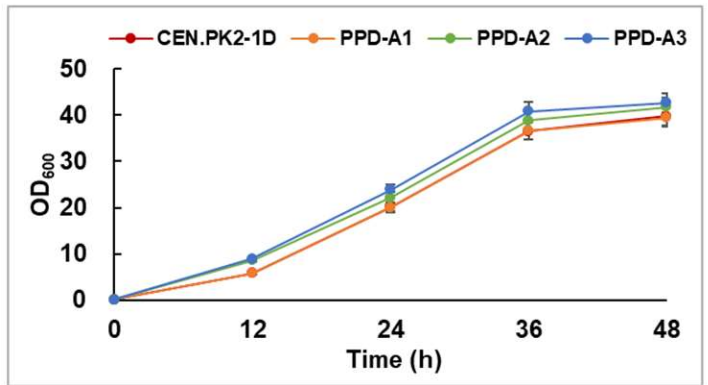

B

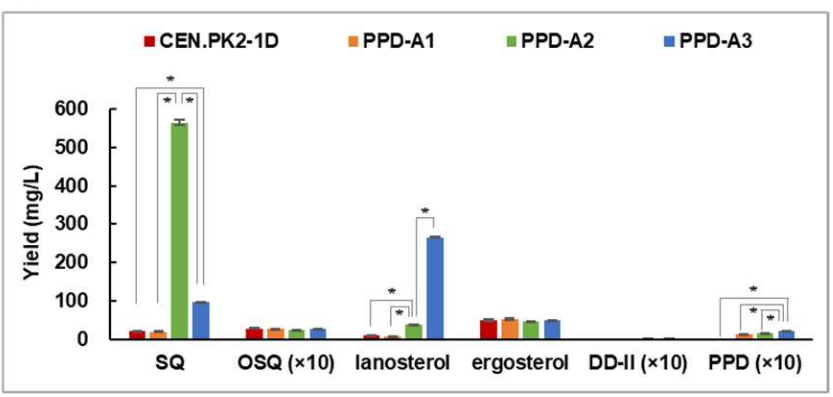

Figure 2. Enhanced production of lanosterol and protopanaxadiol (PPD) in metabolically engineered Saccharomyces cerevisiae strains. (A) Cell growth curves of engineered strains during flask culture in YPD medium. (B) Production of squalene (SQ), 2,3-oxidosqualene (OSQ), lanosterol, ergosterol, dammarenediol-II (DD-II), and PPD in engineered strains at $48 \mathrm{~h}$. Data are presented as means with standard deviation of biological triplicates. Statistical analysis was performed using Student's $t$-test $\left({ }^{*}, p<0.05\right)$. 


\subsection{Enhancing the MVA Pathway and Squalene Monooxygenase Stability Upregulated the Triterpene Biosynthetic Pathway}

Enhancing MVA-pathway metabolic flux is an efficient way to improve triterpene production in yeast $[7,11,31-33]$. We used tHMGR1-overexpression to improve PPD production (Figure 1). We constructed the tHMGR1 expression cassette controlled by the GPD promoter and integrated it into the PPD-A1 strain, producing the PPD-A2 strain (Table 1), which was then subjected to shake-flask fermentation. However, the PPD production of PPD-A2 was not better than that of PPD-A1, although PPD-A2 produced substantially more squalene, at $564.4 \mathrm{mg} / \mathrm{L}$ (Figure 2). PPD-A2 produced more lanosterol, at $38.6 \mathrm{mg} / \mathrm{L}$, but did not differ from PPD-A1 in 2,3-oxidosqualene and ergosterol production. Therefore, tHMGR1-overexpression effectively increased the production of squalene and lanosterol, but not of PPD.

To convert more of the accumulated squalene into 2,3-oxidosqualene, it is necessary to overexpress ERG1. ERG1 is a key regulator of sterol homeostasis [18,34]. In yeast, excess lanosterol accumulation leads to ERG1 degradation via the ER-associated protein degradation (ERAD) pathway [34] found that ERG1 was a target of the ERAD-associated protein ubiquitin ligase Doa10, whereas an ERG1 derivate (K278R/K284R/K311R/K360R) was stabilized from ERAD-mediated protein degradation. Here, we chose to overexpress this ERG1-derivate (ERG1m hereafter), rather than the native ERG1, in the PPD-A2 strain. To do this, we constructed the ERG1m expression cassette controlled by the TEF1 promoter and integrated it into the PPD-A2 strain, producing the PPD-A3 strain. Following shake-flask fermentation of PPD-A3, squalene production was indeed lower in PPD-A3, at $96.1 \mathrm{mg} / \mathrm{L}$, than in PPD-A2 (Figure 2), and PPD production was higher, at $2.2 \mathrm{mg} / \mathrm{L}$. Notably, lanosterol production was also higher in PPD-A3, at $265.8 \mathrm{mg} / \mathrm{L}$, whereas that of ergosterol was similar, relative to that of PPD-A2. Therefore, enhancing the MVA pathway via tHMGR1 overexpression and increasing ERG1 stability via ERG1m overexpression improved the triterpene biosynthetic pathway and particularly, lanosterol and PPD production.

\subsection{CRISPRi-Mediated ERG7 Suppression Improved PPD Production}

Although PPD-A3 produced more PPD than PPD-A2, lanosterol showed greater fold change than PPD from PPD-A2 to PPD-A3, suggesting that, in PPD-A3, most of the 2,3oxidosqualene was converted to lanosterol rather than DD-II and PPD. To further improve PPD production in engineered yeast strains, it is necessary to efficiently suppress lanosterol biosynthesis by quantitative suppression of ERG7 expression. Partial suppression of ERG7 expression using the methionine-suppressible MET3 promoter or antisense ERG7 fragment has been reported [23,31,35]. However, MET3 promoter-mediated ERG7 suppression requires extra methionine to continuously suppress $E R G 7$ expression. Further, antisense ERG7 fragment-mediated ERG7 suppression did not quantitatively suppress ERG7 expression. In addition, the long antisense RNA involved might be unstable. To address these limitations, we used the CRISPRi system to constitutively and quantitatively suppress $E R G 7$ expression. We first designed five ERG7 promoter-targeting sgRNAs (sgRNA1-5) using an online tool (https://lp2.github.io/yeast-crispri/, accessed 1 December 2017) (Figure 3). We then synthesized five SNR52pro-sgRNA-SUP4ter cassettes and cloned them into the dCas9-expressing plasmid pTDH3-dCas9-Mxi1. To co-express the dCas9 and sgRNA in yeast, we constructed five dCas9-sgRNA cassettes and integrated them into the PPD-A3 strain, producing strains PPD-A3-sgRNA1-5. To determine whether the dCas9-sgRNA cassettes suppress ERG7 expression and improve PPD production in yeast, we performed shake-flask fermentation of strains PPD-A3 and PPD-A3-sgRNA1-5 in YPD medium for $48 \mathrm{~h}$ (Figures 3 and 4). We first analyzed the ERG7 expression levels of these strains using quantitative RT-PCR (Figure 3). Relative to the expression in the control strain (PPD-A3), the ERG7 expression levels of strains PPD-A3-sgRNA1-5 were decreased 0.73-, 0.58-, 0.24-, 0.40-, and 0.66-fold, respectively. PPD-A3-sgRNA3 exhibited the most effective ERG7 suppression. To exclude the possibility that dCas9 expression differed among strains PPD-A3-sgRNA1-5, we analyzed their dCas9 expression using quantitative 
RT-PCR (Supplementary Figure S1); dCas9 expression was similar among these strains, suggesting that their different $E R G 7$ expression resulted from different sgRNA efficiencies.

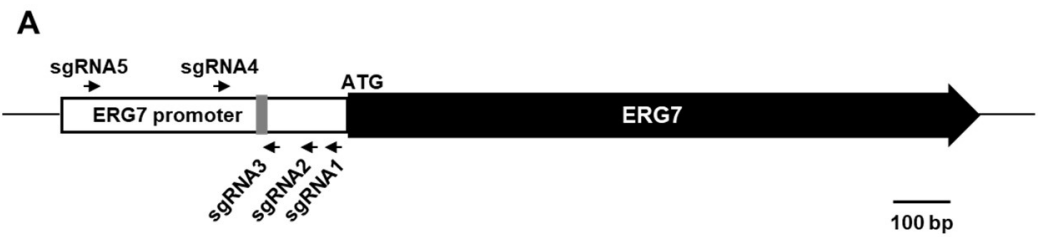

B

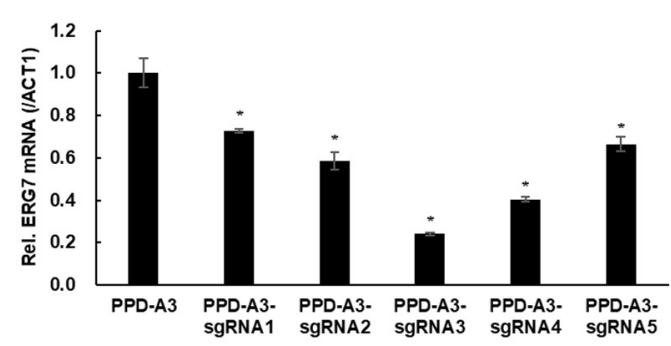

Figure 3. CRISPRi-mediated ERG7 suppression in the protopanaxadiol (PPD)-A3 strain. (A) Schematic of ERG7 promoter-targeting sgRNAs. The arrowhead of each sgRNA indicates the sgRNA direction. The gray vertical bar in the ERG7 promoter indicates the TATA-box. (B) Relative ERG7 mRNA levels in engineered strains cultivated for $48 \mathrm{~h}$ in YPD medium. Data are presented as means with standard deviation of biological triplicates. Statistical analysis was performed using Student's $t$-test $(*, p<0.05)$.

A

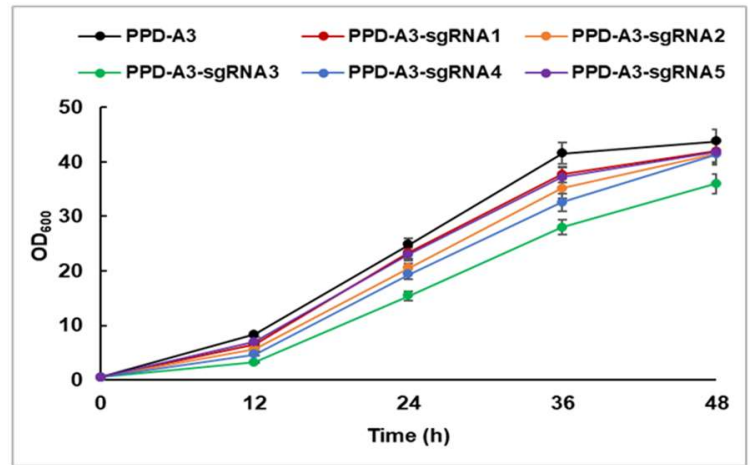

B

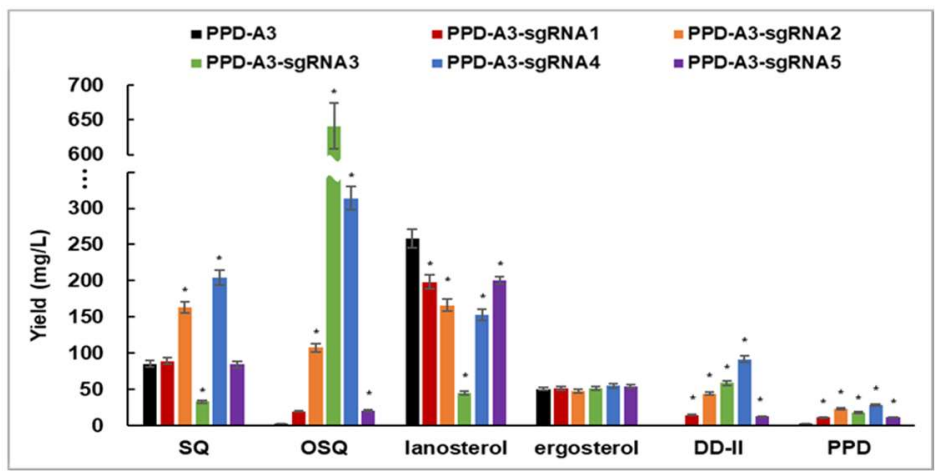

Figure 4. Enhanced production of protopanaxadiol (PPD) by dCas9-sgRNA cassettes in metabolically engineered Saccharomyces cerevisiae strains. (A) Cell growth curves of engineered strains during flask culture in YPD medium. (B) Production of squalene (SQ), 2,3-oxidosqualene (OSQ), lanosterol, ergosterol, dammarenediol-II (DD-II), and PPD in engineered strains at $48 \mathrm{~h}$. Data are presented as means with standard deviation of biological triplicates. Statistical analysis was performed using Student's $t$-test $\left.{ }^{*}, p<0.05\right)$.

The cell growth of PPD-A3-sgRNA1-5 was lower than that of PPD-A3 (Figure 4A and Supplementary Table S4). In particular, PPD-A3-sgRNA3 exhibited severe growth retardation, suggesting that excessive ERG7 suppression might damage cell viability. To determine whether ERG7 suppression by dCas9-sgRNAs resulted in the suppression of lanosterol biosynthesis and improvement of PPD production, we analyzed lanosterol and PPD production after shake-flask fermentation, using the PPD-A3 and PPD-A3-sgRNA1-5 strains (Figure 4B). Lanosterol production in PPD-A3 was $258.6 \mathrm{mg} / \mathrm{L}$, and was lower, at $198.2,165.9,44.8,152.7$, and $200.8 \mathrm{mg} / \mathrm{L}$, respectively, in PPD-A3-sgRNA1-5. Notably, the order of the suppression efficiency of lanosterol biosynthesis in strains PPD-A3-sgRNA1-5 was the same as that for ERG7 expression, suggesting that dCas9-sgRNA could quanti- 
tatively suppress both ERG7 expression and lanosterol biosynthesis. PPD production by PPD-A3 was $1.9 \mathrm{mg} / \mathrm{L}$, and was higher, at 11.2, 23.0, 17.8, 27.6, and $11.1 \mathrm{mg} / \mathrm{L}$, respectively, in PPD-A3-sgRNA1-5. Notably, PPD-A3-sgRNA3, which exhibited the strongest ERG7 suppression, did not exhibit the highest PPD production. Rather, PPD-A3-sgRNA4 exhibited the highest PPD production, suggesting that excessive ERG7 suppression might disadvantage the biosynthetic pathway of the secondary metabolite. This indicates that our method efficiently suppressed ERG7 expression, thereby improving PPD production in the engineered yeast strain.

\subsection{PPD Production via Batch-Fed Fermentation}

Although several studies have assessed the use of CRISPRi to regulate metabolic flux in yeast, most of them have been limited to a few days in duration [36-39]. To determine whether dCas9-sgRNA-mediated ERG7 suppression was effective over longer periods, we performed batch-fed fermentation, using the PPD-A3 and PPD-A3-sgRNA4 strains, in a 5-L bioreactor for $216 \mathrm{~h}$; the strains exhibited similar cell growth profiles (Figure 5A). Lanosterol production by PPD-A3 reached $541.5 \mathrm{mg} / \mathrm{L}$ at $120 \mathrm{~h}$ and maintained this until $216 \mathrm{~h}$ (Figure 5B). For PPD-A3-sgRNA4, however, lanosterol production reached $210 \mathrm{mg} / \mathrm{L}$ at $120 \mathrm{~h}$, then decreased to $140 \mathrm{mg} / \mathrm{L}$ at $216 \mathrm{~h}$ (Figure 5B). At $216 \mathrm{~h}$, PPD production by PPDA3 and PPD-A3-sgRNA4 was $52.1 \mathrm{mg} / \mathrm{L}$ and $294.5 \mathrm{mg} / \mathrm{L}$, respectively (Figure 5C). This indicates that dCas9-sgRNA-mediated ERG7 suppression over longer periods effectively suppressed lanosterol biosynthesis and improved PPD production in the engineered strain.

A

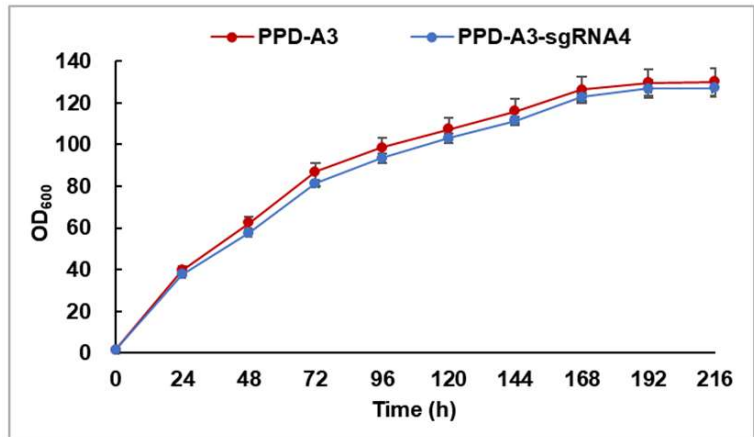

C

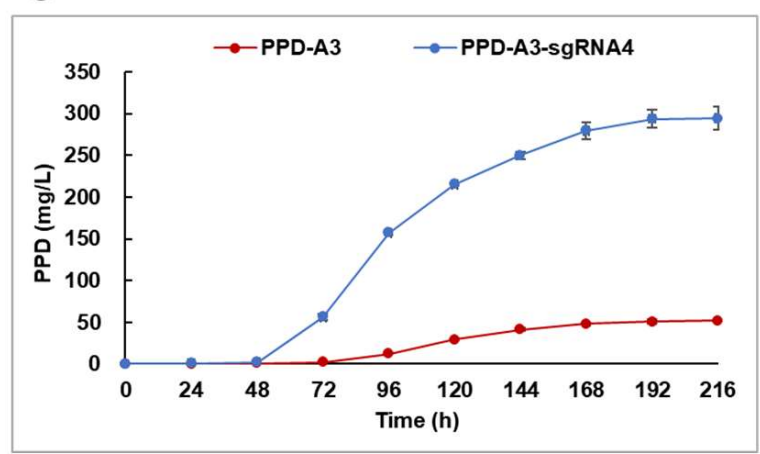

\section{B}

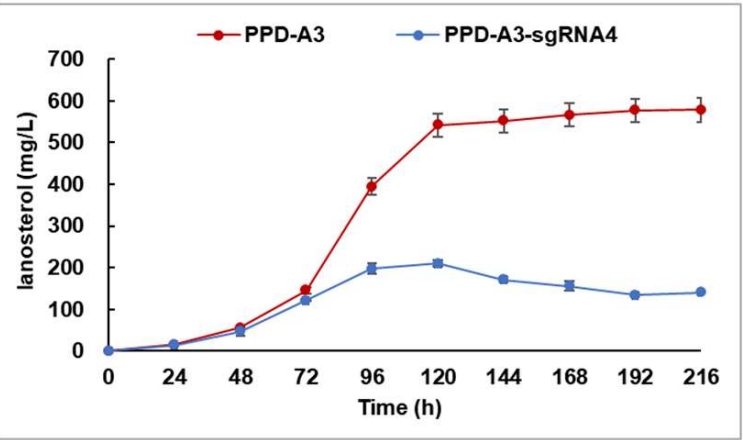

Figure 5. Batch-fed fermentation of protopanaxadiol (PPD)-A3 and PPD-A3-sgRNA4 strains in $5 \mathrm{~L}$ fermenter. (A) Cell growth curves during batch-fed fermentation. Production of (B) lanosterol and (C) PPD during batch-fed fermentation.

\section{Discussion}

PPD has important pharmacological properties, including anti-cancer activity. To improve production of this high-value-added product, we metabolically engineered yeast strains to biosynthesize it more efficiently. We first constructed a PPD-producing yeast strain in which tHMGR1 was overexpressed to enhance the MVA pathway. Next, we 
overexpressed the ERG1m gene in this engineered strain, to enhance squalene conversion to 2,3-oxidosqualene. In spite of this, PPD production remained low, because the PPD and native ergosterol biosynthetic pathways compete for 2,3-oxidosqualene. To overcome this competition, we established a CRISPRi-based metabolic engineering strategy, and achieved stable, sustained, high-level PPD production in S. cerevisiae.

In S. cerevisiae, HMGR1 and ERG1 are key regulatory enzymes in the sterol biosynthetic pathway $[34,40]$. They are regulated by negative feedback at transcriptional and protein levels, to maintain ergosterol homeostasis. When we overexpressed tHMGR1 in the PPD-producing yeast strain, squalene accumulated, whereas 2,3-oxidosqualene did not accumulate, suggesting that ERG1 is also a rate-limiting enzyme in the sterol biosynthetic pathway. When we further overexpressed the ERG1m gene in the PPD-producing strain and most of the squalene was converted to lanosterol and PPD. However, lanosterol production far exceeded PPD production, suggesting that most of the 2,3-oxidosqualene were converted to lanosterol rather than DD-II and PPD. We propose a possible reason for thisthe enzymatic activity of ERG7 might be stronger than that of PgDS. In humans, lanosterol synthase is a monotopic endoplasmic reticulum (ER)-membrane protein comprising two $(\alpha / \alpha)$ barrel domains connected by loops, and three smaller $\beta$-structures [41]. The active site cavity is located in the center of the enzyme, and 2,3-oxidosqualene enters this active site cavity. Although PgDS also functions at the ER, it does not contain a transmembrane domain [42]. Thus, ERG7 might have a competitive advantage over PgDS in substrate binding. To overcome this competition, we decided to suppress the ERG7 expression in engineered yeast strains.

To suppress ERG7 expression, we applied CRISPRi-guided regulation of ERG7 expression, using dCas9 and five ERG7 promoter-targeting sgRNAs (sgRNA1-5). Since the stable expression of dCas9 and sgRNA is important for constitutive suppression of target gene expression, we integrated dCas9-sgRNA cassettes into chromosomes of the PPD-A3 strain. dCas9 expression was similar among these strains, whereas ERG7 expression differed substantially. The efficiency of the sgRNAs that we constructed for ERG7 suppression was ranked (in decreasing order), as follows: sgRNA3, sgRNA4, sgRNA2, sgRNA5, and sgRNA1. Notably, the dCas9-sgRNA3 cassette retarded cell growth, suggesting that there might be an ERG7 expression threshold that affects cell viability. It is possible, by using different sgRNAs targeting the same gene, to reveal differences in efficiency [37-39,42]. It is likely that sgRNA3 was the most effective at suppressing ERG7 expression because its target region is closest to the TATA-box in the ERG7 promoter. Indeed, sgRNA1 and sgRNA5, for which the target regions are distant from the TATA-box in the ERG7 promoter, were less effective at suppressing ERG7 expression. These findings indicate that the sgRNA target site is a key aspect in the quantitative regulation of gene expression. Further, with the exception of ERG7 suppression by sgRNA3, the relative differences in the efficiency of the constructed sgRNAs were consistent with those in PPD production. This indicates that CRISPRi-guided suppression of ERG7 expression suppressed lanosterol biosynthesis and improved PPD production.

Notably, CRISPRi-guided suppression of ERG7 resulted in the accumulation of squalene and 2,3-oxidosqualene, but not ergosterol. Yeast cells not only regulate sterol biosynthesis but also convert excessive sterols into steryl esters, which are then stored in lipid droplets or secreted into the extracellular matrix [18]. Considering that overexpression of $t H M G R 1$ and EGR1m enhanced sterol biosynthesis by inhibiting negative feedback, accumulation of squalene and 2,3-oxidosqualene indicates that CRISPRi-guided ERG7 suppression is a powerful tool for regulating metabolic flux. To further enhance the conversion of 2,3-oxidosqualene to DD-II and PPD in yeast, it might be helpful to increase PgDS, $P g P P D S$, and $P g C P R$ expression.

The stability of the dCas9-sgRNA complex, and the accuracy of sgRNA-targeting, are important factors for achieving stable and efficient regulation of target gene expression over longer periods, when using CRISPRi to bioengineer yeast strains. Most previous studies on this have been conducted over a few days, possibly because of difficulties in 
maintaining dCas9-sgRNA complex stability [37-39,42]. Here, we performed batch-fed fermentation over 9 days, using the PPD-A3 and PPD-A3-sgRNA4 strains, to confirm the effect of CRISPRi-guided ERG7 suppression over longer periods. At $216 \mathrm{~h}$, lanosterol production was lower, but PPD production was higher in the PPD-A3-sgRNA4 strain than in the unmodified PPD-producing (control) strain. This is the first study to demonstrate the efficacy of CRISPRi-guided regulation of metabolic flux for longer periods. These findings may help in the metabolic engineering of industrial strains to improve production of target molecules.

\section{Materials and Methods}

\subsection{Strains and Medium}

Saccharomyces cerevisiae CEN.PK2-1D was obtained from EUROSCARF (http:/ / www. euroscarf.de/) and used as a parent strain for all engineered yeast strains. The yeast strains are listed in Table 1. Constructed yeast strains were grown in YPD medium $(10 \mathrm{~g} / \mathrm{L}$ yeast extract, $20 \mathrm{~g} / \mathrm{L}$ peptone, and $50 \mathrm{~g} / \mathrm{L}$ glucose) or SD medium $(6.7 \mathrm{~g} / \mathrm{L}$ yeast nitrogen base and $50 \mathrm{~g} / \mathrm{L}$ glucose) at $26{ }^{\circ} \mathrm{C}$, with shaking at $220 \mathrm{rpm}$, lacking histidine, tryptophan, leucine, and uracil where appropriate.

Escherichia coli DH5 $\alpha$ (Enzynomics, Republic of Korea) was used for transformation and plasmid amplification. Escherichia coli cells were grown in Luria-Bertani (LB) medium $(10 \mathrm{~g} / \mathrm{L}$ tryptone, $5 \mathrm{~g} / \mathrm{L}$ yeast extract, and $10 \mathrm{~g} / \mathrm{L} \mathrm{NaCl})$ with $100 \mathrm{mg} / \mathrm{L}$ ampicillin at $37^{\circ} \mathrm{C}$, with shaking at $200 \mathrm{rpm}$.

\subsection{Plasmid Construction}

Yeast expression plasmids pRS424TEF1(\#87365), pRS426GPD(\#87361), pRS426PGK1(\#1370), Cas9-NAT(\#64329), and pTDH3-dCas9-Mxi1(\#46921) and sgRNA-expressing plasmid pRS42H(\#64330) were purchased from Addgene (Cambridge, MA, USA), and sgRNAexpressing plasmid pRS42K(P30637) was purchased from EUROSCARF (Frankfurt, Germany). Dammarenediol-II synthase (PgDS; GenBank: AB265170), PPD synthase (PgPPDS; GenBank: JN604537), and cytochrome P450 reductase (PgCPR; GenBank: KF486915), each with the BamHI and XhoI restriction sites at their $5^{\prime}$ - and $3^{\prime}$ - ends, respectively, were synthesized with codon optimization for expression in S. cerevisiae (BIONEER, Daejeon, Korea). $P g D S$ and $P g P P D S$ were cloned into the pRS426GPD vector, and $P g C P R$ was cloned into pRS426PGK1. For the CRISPR/Cas9-guided integration of the PgDS, PgPPDS, and PgCPR genes, sgRNAs targeting downstream of TEF2, upstream of GLK1, and upstream of RPS17B were designed using an online tool (https:/ /www.atum.bio/eCommerce/cas9/input, Accessed 2 July 2018). All integration sites in this study were previously chosen in our laboratory. TEF2- and GLK1-targeting sgRNAs were cloned into the pRS42K vector, and the RPS17B-targeting sgRNA was cloned into the pRS42H vector. The N-terminally truncated HMG-CoA reductase 1 ( $t H M G R 1$ gene), with the BamHI and XhoI restriction sites at its $5^{\prime}$ and $3^{\prime}$ - ends, respectively, was amplified from yeast genomic DNA and cloned into the pRS426GPD vector. The EGR1m gene, in which four lysine residues of the yeast ERG1 gene had been replaced with arginine residues (K278R, K284R, K311R, and K360R) in a previous study (Foresti et al., 2013), with the BamHI and XhoI restriction sites at its $5^{\prime}$ - and $3^{\prime}$ - ends, respectively, was synthesized with codon optimization (BIONEER), and was cloned into the pRS424TEF1 vector. For CRISPRi-mediated ERG7 suppression, ERG7 promoter-targeting sgRNAs were designed using an online tool (https://lp2.github.io/yeast-crispri/, Accessed 2 July 2018) (Supplementary Table S1). To construct the dCas9/sgRNA co-expression plasmids (pTDH3-dCas9-sgRNA1-5), SNR52pro-sgRNA(1-5)-SUP4ter cassettes, each with the EcoRI and SpeI restriction sites at their $5^{\prime}$ - and $3^{\prime}$ - ends, respectively, were synthesized (BIONEER) and cloned into pTDH3-dCas9-Mxi1. The plasmids and primers used are listed in Supplementary Tables S2 and S3, respectively. 


\subsection{Engineering S. cerevisiae for PPD Production}

Saccharomyces cerevisiae strain CEN.PK2-1D was transformed using the Alkali-Cation Yeast Transformation Kit (\#112200200, MP Biomedicals, Solon, OH, USA), according to manufacturer's protocol. The PPD-A1 strain was constructed by integrating the PgDS, $P g P P D S$, and $P g C P R$ genes downstream of TEF2, upstream of GLK1, and upstream of $R P S 17 B$, respectively, in the CEN.PK2-1D strain.

The GPDpro-PgDS-CYC1ter cassette, with the homologous recombination region of the partial TEF2 site, was amplified from the PgDS-carrying pRS426GPD vector, using primer set TEF2-Integ-F/TEF2-Ineg-R. This integration cassette, with plasmids Cas9-NAT and pRS42K-sgRNA (TEF2), was co-transformed into strain CEN.PK2-1D, followed by selection on a YPD/ClonNAT/G418 plate. Strains were verified by diagnostic PCR, and colonies containing the desired plasmids and integration cassette were cultivated at $30^{\circ} \mathrm{C}$ in YPD/ClonNAT medium for $24 \mathrm{~h}$ to remove pRS42K-sgRNA (TEF2), producing the CEN.PK2-1D-PgDS-Cas9-NAT strain.

The GPD pro-PgPPDS-CYC1ter cassette, with the homologous recombination region of the partial GLK1 site, was amplified from the PgPPDS-carrying pRS426GPD vector using the primer set GLK1-Integ-F/GLK1-Integ-R. This integration cassette, with the plasmid and pRS42H-sgRNA (GLK1), was co-transformed into strain CEN.PK2-1D-Cas9-NAT-PgDS, followed by selection on a YPD/ClonNAT/Hygromycin B plate. Strains were verified with diagnostic $\mathrm{PCR}$, and colonies containing the desired plasmids and integration cassette were cultivated at $30{ }^{\circ} \mathrm{C}$ in YPD/ClonNAT medium for $24 \mathrm{~h}$ to remove pRS42H-sgRNA (GLK1), producing the CEN.PK2-1D-Cas9-NAT-PgDS-PgPPDS strain.

The PGK1pro-PgCPR-CYC1ter cassette, with the homologous recombination region of the partial $R P S 17 B$ site, was amplified from the $P g C P R$-carrying pRS426PGK1 vector, using the primer set RPS17B-Integ-F/RPS17B-Integ-R. This integration cassette, with plasmid pRS42K-sgRNA (RPS17B), was co-transformed into strain CEN.PK2-1D-Cas9-NAT-PgDSPgPPDS, followed by selection on a YPD/ClonNAT/G418 plate. Strains were verified by diagnostic $\mathrm{PCR}$, and colonies containing the desired plasmids and integration cassette were cultivated at $30{ }^{\circ} \mathrm{C}$ in YPD medium for $24 \mathrm{~h}$ to remove Cas9-NAT and pRS42K-sgRNA $(R P S 17 B)$, producing the PPD-A1 strain.

Strain PPD-A2 was constructed by integrating the $t H M G 1$ gene downstream of the TCB2 site of PPD-A1. The [GPDpro-tHMG1-CYC1ter]-URA3 cassette, with the homologous recombination region of the partial TCB2 site, was amplified from the $t H M G 1$-carrying pRS426GPD vector, using the primer set TCB2-Integ-F/TCB2-Integ-R. This integration cassette was transformed into strain PPD-A1, followed by selection on an SD/-URA plate. Strains were verified by diagnostic $\mathrm{PCR}$, and colonies containing the integration cassette were cultivated at $30^{\circ} \mathrm{C}$ in SD/-URA medium, producing the PPD-A2 strain.

Strain PPD-A3 was constructed by integrating the ERG1m gene into the trp1-289 site of PPD-A2. The [TEF1pro-ERG1m-CYC1ter]-TRP1 cassette, with the homologous recombination region of the partial TRP1 gene, was amplified from the ERG1m-carrying pRS424TEF1 vector, using the primer set TRP1-Integ-F/TRP1-Integ-R. This integration cassette was transformed into strain PPD-A2, followed by selection on an SD/-URA/-TRP plate. Strains were verified by diagnostic PCR, and colonies containing integration cassette were cultivated at $30{ }^{\circ} \mathrm{C}$ in SD/-URA/-TRP medium, producing the PPD-A3 strain.

Strains PPD-A3-sgRNA1-5 were constructed by integrating the dCas9 and ERG7 promoter-targeting sgRNA genes upstream of the ISR1 site of PPD-A3. The dCas9[SNR52pro-sgRNA-SUP4ter]-LEU2 cassettes, with the homologous recombination region of the partial ISR1 site, were amplified from pTDH3-dCas9-Mxi1-sgRNA1-5 using the primer set ISR1-Integ-F/ISR1-Integ-R. Each integration cassette was transformed into PPDA3, followed by selection on an SD/-URA/-TRP/-LEU plate. Strains were verified by diagnostic PCR, and colonies containing the integration cassette were cultivated at $30{ }^{\circ} \mathrm{C}$ in SD/-URA/-TRP/-LEU medium, producing strains PPD-A3-sgRNA1-5. 


\subsection{Yeast Cultivation and Batch-Fed Fermentation}

For shake-flask fermentation, YPD medium was used to cultivate the yeast strains. First, $1 \mathrm{~mL}$ of stock cells in $25 \%$ glycerol was inoculated into a $250 \mathrm{~mL}$ baffled flask (TriForest, Irvine, CA, USA) containing $29 \mathrm{~mL}$ of YPD medium and cultivated at $26{ }^{\circ} \mathrm{C}$, with shaking at $220 \mathrm{rpm}$, to an optical density at $600 \mathrm{~nm}$ (OD600) of approximately 6.0 which was measured by a GENESYS 20 visible spectrophotometer (Thermo Scientific, Waltham, MA, USA). Then, $1 \mathrm{~mL}$ of seed culture was inoculated into a $250 \mathrm{~mL}$ baffled flask containing $29 \mathrm{~mL}$ of YPD medium and cultivated at $26{ }^{\circ} \mathrm{C}$, with shaking at $220 \mathrm{rpm}$, for $48 \mathrm{~h}$. Flask-fermentation results are presented as means with standard deviation based on biological triplicates.

Strains PPD-A3 and PPD-A3-sgRNA4 were used for the production of PPD via batchfed fermentation in a stirred glass tank $5 \mathrm{~L}$ bioreactor (CNS, Daejeon, Korea), with an initial working volume of $2 \mathrm{~L}$ YPD medium. Seed culture was prepared in two steps. First, $1 \mathrm{~mL}$ of stock cells in $25 \%$ glycerol was inoculated into a $250 \mathrm{~mL}$ baffled flask containing $29 \mathrm{~mL}$ of YPD medium, followed by cultivation at $26^{\circ} \mathrm{C}$ and shaking at $220 \mathrm{rpm}$, to an OD600 of approximately 6.0. Then, $12 \mathrm{~mL}$ of the first seed culture was inoculated into two $1 \mathrm{~L}$ baffled flasks (Duran) containing $138 \mathrm{~mL}$ of YPD medium, and cultivated at $26^{\circ} \mathrm{C}$ with shaking at $220 \mathrm{rpm}$, to an OD600 of approximately 7.0. Then, $300 \mathrm{~mL}$ of the second seed culture was inoculated into the $5 \mathrm{~L}$ bioreactor containing $1.7 \mathrm{~L}$ of YPD medium. Fermentation was carried out at $26^{\circ} \mathrm{C}$ with shaking at $300 \mathrm{rpm}$ and air flow at $4 \mathrm{~L} / \mathrm{min}$. $\mathrm{pH}$ was controlled at 5.5 by automatic addition of $15 \%$ ammonium hydroxide $(v / v)$. To minimize foaming in the bioreactor, $10 \%$ Antifoam $204(v / v)$ was used. After the initial glucose was completely consumed, a solution containing $500 \mathrm{~g} / \mathrm{L}$ glucose, $18.7 \mathrm{~g} / \mathrm{L} \mathrm{KH}_{2} \mathrm{PO}_{4}, 6.5 \mathrm{~g} / \mathrm{L} \mathrm{K}_{2} \mathrm{SO}_{4}$, $0.53 \mathrm{~g} / \mathrm{L} \mathrm{Na}_{2} \mathrm{SO}_{4}, 9.75 \mathrm{~g} / \mathrm{L} \mathrm{MgSO} 4 \cdot 7 \mathrm{H}_{2} \mathrm{O}, 10 \mathrm{~g} / \mathrm{L}$ histidine, $10 \mathrm{~g} / \mathrm{L}$ leucine, $10 \mathrm{~mL} / \mathrm{L}$ of trace metal solution, and $12 \mathrm{~mL} / \mathrm{L}$ of vitamin solution [23], was added to the bioreactor, and the dissolved oxygen level was increased to above $50 \%$. The glucose concentration in the bioreactor was maintained below $10 \mathrm{~g} / \mathrm{L}$.

\subsection{Metabolite Extraction and HPLC Analysis}

Yeast cells at a density equivalent to an OD600 of 40 were collected from the flaskfermentation and batch-fed fermentation processes into $2 \mathrm{~mL}$ Safe-Lock Eppendorf tubes, and centrifuged at $13,000 \times g$ for $5 \mathrm{~min}$. The supernatant was discarded, and the collected cells were resuspended in $1 \mathrm{~mL}$ of a methanol-acetone mixture $(1: 1 \mathrm{v} / v)$. The cells were then lysed using an MM400 homogenizer (Retsch, Germany), according to the manufacturer's protocol. Samples were then centrifuged at $13,000 \times g$ for $10 \mathrm{~min}$. The supernatant $(30 \mu \mathrm{L})$ was then injected into an Agilent 1260 Infinity II HPLC system (Agilent, Santa Clara, CA, USA), with UV detection at $203 \mathrm{~nm}$. Chromatographic separation was conducted using a Prodigy $5 \mu \mathrm{m}$ ODS-2 LC column $(4.6 \mathrm{~mm} \times 150 \mathrm{~mm}$, Phenomenex, Torrance, CA, USA). The mobile phase consisted of water (A) and acetonitrile (B), using a gradient program of $32-65 \%$ B at $0-8 \mathrm{~min}, 65-90 \%$ B at $8-12 \mathrm{~min}, 90 \%$ B at $12-20 \mathrm{~min}, 90-100 \%$ $\mathrm{B}$ at $20-30 \mathrm{~min}, 100 \% \mathrm{~B}$ at $30-65 \mathrm{~min}, 100-32 \% \mathrm{~B}$ at $65-66 \mathrm{~min}$, and $32 \% \mathrm{~B}$ at $66-70 \mathrm{~min}$. The solvent flow rate was $1.0 \mathrm{~mL} / \mathrm{min}$ and the column temperature was set to $30^{\circ} \mathrm{C}$. Analytical grade squalene, 2,3-oxidosqualene, lanosterol, and ergosterol were purchased from Sigma-Aldrich (Burlington, MA, USA). Dammarenediol-II and PPD were purchased from ChemFaces (Wuhan, China).

\subsection{RNA Extraction and $q R T-P C R$}

Yeast cells at a density equivalent to an OD600 of 20 were collected from the flaskfermentation process into a $2 \mathrm{~mL}$ Safe-Lock Eppendorf tube and centrifuged at 13,000 $\times g$ for 5 min. Total RNA was extracted using the RNeasy Mini Kit (Qiagen, Germany), and cDNA was obtained using M-MLV Reverse Transcriptase (Thermo Scientific, Waltham, MA, USA), both according to the manufacturers' protocol. The relative mRNA level of each gene was determined using real-time PCR (Bio-Rad, Hercules, CA, USA), using specific primer sets (Supplementary Table S3), with ACTIN1 as the reference gene. 
Supplementary Materials: The following are available online at https://www.mdpi.com/article/10 .3390/ijms222111836/s1.

Author Contributions: Conceptualization, S.-H.L., S.-C.K. and J.-I.B.; methodology, S.-H.L., J.-I.B., J.-H.L., J.-W.S. and B.-M.J.; software, S.-H.L., J.-I.B., M.-S.K. and B.-M.J.; validation, S.-H.L., J.I.B., S.-C.K., J.-H.L. and B.-M.J.; formal analysis, S.-H.L., J.-I.B., S.-H.P., S.-J.K., S.-C.K. and B.-M.J.; investigation, S.-H.L., J.-I.B., S.-H.P., S.-J.K., J.-Y.L., J.-W.S., S.-C.K. and B.-M.J.; resources, S.-H.L., J.-I.B., J.-Y.B. and B.-M.J.; data curation, S.-H.L. and J.-I.B.; writing-original draft preparation, S.-H.L. and J.-I.B.; writing-review and editing, J.-I.B. and B.-M.J.; visualization, S.-H.L., J.-I.B. and B.-M.J.; supervision, S.-C.K.; project administration, S.-C.K.; funding acquisition, S.-C.K. All authors have read and agreed to the published version of the manuscript.

Funding: This work was supported by the Intelligent Synthetic Biology Center of the Global Frontier Project, funded by the Ministry of Education, Science, and Technology (grant number 2011-0031955, Korea; the Bio-Synergy Research Project (grant number NRF-2021M3A9C4001028) of the Ministry of Science, ICT, and Future Planning through the National Research Foundation, Advancement of Active Biomedical material Project, funded by the Ministry of Trade (grant number P0014633), Korea; and the Development of Industrialization Technology for Crop virus and Pest Project, funded by the Ministry of Agriculture, Food, and Rural Affairs (grant number 321109041SB010), Korea. The funding agencies had no role in study design; in the collection, analysis and interpretation of data; in the writing of the report; or in the decision to submit the article for publication.

Conflicts of Interest: The authors declare that they have no conflict of interest.

\section{References}

1. Siraj, F.M.; SathishKumar, N.; Kim, Y.J.; Kim, S.Y.; Yang, D.C. Ginsenoside F2 possesses anti-obesity activity via binding with PPARgamma and inhibiting adipocyte differentiation in the 3T3-L1 cell line. J. Enzyme Inhib. Med. Chem. 2015, 30, 9-14. [CrossRef] [PubMed]

2. Kim, D.E.; Chang, B.Y.; Jeon, B.M.; Baek, J.I.; Kim, S.C.; Kim, S.Y. SGL 121 Attenuates Nonalcoholic Fatty Liver Disease through Adjusting Lipid Metabolism Through AMPK Signaling Pathway. Int. J. Mol. Sci. 2020, 21, 4534. [CrossRef]

3. Park, S.; Ko, E.; Lee, J.H.; Song, Y.; Cui, C.-H.; Hou, J.; Jeon, B.M.; Kim, H.S.; Kim, S.C. Gypenoside LXXV Promotes Cutaneous Wound Healing In Vivo by Enhancing Connective Tissue Growth Factor Levels Via the Glucocorticoid Receptor Pathway. Molecules 2019, 24, 1595. [CrossRef]

4. Li, X.; Chu, S.; Lin, M.; Gao, Y.; Liu, Y.; Yang, S.; Zhou, X.; Zhang, Y.; Hu, Y.; Wang, H.; et al. Anticancer property of ginsenoside Rh2 from ginseng. Eur. J. Med. Chem. 2020, 203, 112627. [CrossRef]

5. Gao, H.; Kang, N.; Hu, C.; Zhang, Z.; Xu, Q.; Liu, Y.; Yang, S. Ginsenoside Rb1 exerts anti-inflammatory effects in vitro and in vivo by modulating toll-like receptor 4 dimerization and NF-kB/MAPKs signaling pathways. Phytomedicine 2020, 69, 153197. [CrossRef]

6. Chen, W.; Balan, P.; Popovich, D.G. Analysis of Ginsenoside Content (Panax ginseng) from Different Regions. Molecules 2019, 24, 3491. [CrossRef]

7. Dai, Z.; Liu, Y.; Zhang, X.; Shi, M.; Wang, B.; Wang, D.; Huang, L.; Zhang, X. Metabolic engineering of Saccharomyces cerevisiae for production of ginsenosides. Metab. Eng. 2013, 20, 146-156. [CrossRef] [PubMed]

8. Kim, J.E.; Jang, I.S.; Son, S.H.; Ko, Y.J.; Cho, B.K.; Kim, S.C.; Lee, J.Y. Tailoring the Saccharomyces cerevisiae endoplasmic reticulum for functional assembly of terpene synthesis pathway. Metab. Eng. 2019, 56, 50-59. [CrossRef] [PubMed]

9. Kim, J.E.; Jang, I.S.; Sung, B.H.; Kim, S.C.; Lee, J.Y. Rerouting of NADPH synthetic pathways for increased protopanaxadiol production in Saccharomyces cerevisiae. Sci. Rep. 2018, 8, 15820. [CrossRef]

10. Zhao, F.; Bai, P.; Liu, T.; Li, D.; Zhang, X.; Lu, W.; Yuan, Y. Optimization of a cytochrome P450 oxidation system for enhancing protopanaxadiol production in Saccharomyces cerevisiae. Biotechnol. Bioeng. 2016, 113, 1787-1795. [CrossRef]

11. Zhao, F.; Bai, P.; Nan, W.; Li, D.; Zhang, C.; Lu, C.; Qi, H.; Lu, W. A modular engineering strategy for high-level production of protopanaxadiol from ethanol by Saccharomyces cerevisiae. AIChE J. 2018, 65, 866-874. [CrossRef]

12. Zhao, F.; Du, Y.; Bai, P.; Liu, J.; Lu, W.; Yuan, Y. Enhancing Saccharomyces cerevisiae reactive oxygen species and ethanol stress tolerance for high-level production of protopanoxadiol. Bioresour. Technol. 2017, 227, 308-316. [CrossRef] [PubMed]

13. Chu, L.L.; Montecillo, J.A.V.; Bae, H. Recent Advances in the Metabolic Engineering of Yeasts for Ginsenoside Biosynthesis. Front. Bioeng. Biotechnol. 2020, 8, 139. [CrossRef]

14. Vranová, E.; Coman, D.; Gruissem, W. Network analysis of the MVA and MEP pathways for isoprenoid synthesis. Annu. Rev. Plant Biol. 2013, 64, 665-700. [CrossRef] [PubMed]

15. Tansakul, P.; Shibuya, M.; Kushiro, T.; Ebizuka, Y. Dammarenediol-II synthase, the first dedicated enzyme for ginsenoside biosynthesis, in Panax ginseng. FEBS Lett. 2006, 580, 5143-5149. [CrossRef] 
16. Han, J.Y.; Hwang, H.S.; Choi, S.W.; Kim, H.J.; Choi, Y.E. Cytochrome P450 CYP716A53v2 catalyzes the formation of protopanaxatriol from protopanaxadiol during ginsenoside biosynthesis in Panax ginseng. Plant Cell Physiol. 2012, 53, 1535-1545. [CrossRef] [PubMed]

17. Yan, X.; Fan, Y.; Wei, W.; Wang, P.; Liu, Q.; Wei, Y.; Zhang, L.; Zhao, G.; Yue, J.; Zhou, Z. Production of bioactive ginsenoside compound $\mathrm{K}$ in metabolically engineered yeast. Cell Res. 2014, 24, 770-773. [CrossRef]

18. Hu, Z.; He, B.; Ma, L.; Sun, Y.; Niu, Y.; Zeng, B. Recent Advances in Ergosterol Biosynthesis and Regulation Mechanisms in Saccharomyces cerevisiae. Indian J. Microbiol. 2017, 57, 270-277. [CrossRef] [PubMed]

19. Yuan, J.; Ching, C.B. Dynamic control of ERG9 expression for improved amorpha-4,11-diene production in Saccharomyces cerevisiae. Microb. Cell Fact. 2015, 14, 38. [CrossRef]

20. Paddon, C.J.; Westfall, P.J.; Pitera, D.J.; Benjamin, K.; Fisher, K.; McPhee, D.J.; Leavell, M.D.; Tai, A.; Main, A.; Eng, D.; et al. High-level semi-synthetic production of the potent antimalarial artemisinin. Nature 2013, 496, 528-532. [CrossRef]

21. Ozaydın, B.; Burd, H.; Lee, T.S.; Keasling, J.D. Carotenoid-based phenotypic screen of the yeast deletion collection reveals new genes with roles in isoprenoid production. Metab. Eng. 2013, 15, 174-183. [CrossRef]

22. Rodriguez, S.; Denby, C.M.; Van Vu, T.; Baidoo, E.E.; Wang, G.; Keasling, J.D. ATP citrate lyase mediated cytosolic acetyl-CoA biosynthesis increases mevalonate production in Saccharomyces cerevisiae. Microb. Cell Fact. 2016, 15, 48. [CrossRef]

23. Jung, S.C.; Kim, W.; Park, S.C.; Jeong, J.; Park, M.K.; Lim, S.; Lee, Y.; Im, W.T.; Lee, J.H.; Choi, G.; et al. Two ginseng UDPglycosyltransferases synthesize ginsenoside Rg3 and Rd. Plant Cell Physiol. 2014, 55, 2177-2188. [CrossRef] [PubMed]

24. Kirby, J.; Romanini, D.W.; Paradise, E.M.; Keasling, J.D. Engineering triterpene production in Saccharomyces cerevisiae-betaamyrin synthase from Artemisia annua. FEBS J. 2008, 275, 1852-1859. [CrossRef]

25. Broker, J.N.; Muller, B.; van Deenen, N.; Prufer, D.; Gronover, C.S. Upregulating the mevalonate pathway and repressing sterol synthesis in Saccharomyces cerevisiae enhances the production of triterpenes. Appl. Microbiol. Biotechnol. 2018, 102, 6923-6934. [CrossRef] [PubMed]

26. Peng, B.; Plan, M.R.; Chrysanthopoulos, P.; Hodson, M.; Nielsen, L.; Vickers, C.E. A squalene synthase protein degradation method for improved sesquiterpene production in Saccharomyces cerevisiae. Metab. Eng. 2016, 39, 209-219. [CrossRef]

27. Scalcinati, G.; Knuf, C.; Partow, S.; Chen, Y.; Maury, J.; Schalk, M.; Daviet, L.; Nielsen, J.; Siewers, V. Dynamic control of gene expression in Saccharomyces cerevisiae engineered for the production of plant sesquitepene alpha-santalene in a fed-batch mode. Metab. Eng. 2012, 14, 91-103. [CrossRef] [PubMed]

28. Chen, Y.; Ho, J.; Shis, D.L.; Gupta, C.; Long, J.; Wagner, D.S.; Ott, W.; Josić, K.; Bennett, M.R. Tuning the dynamic range of bacterial promoters regulated by ligand-inducible transcription factors. Nat. Commun. 2018, 9, 64. [CrossRef]

29. Lv, L.; Ren, Y.; Chen, J.-C.; Wu, Q.; Chen, G.-Q. Application of CRISPRi for prokaryotic metabolic engineering involving multiple genes, a case study: Controllable P(3HB-co-4HB) biosynthesis. Metab. Eng. 2015, 29, 160-168. [CrossRef] [PubMed]

30. Yasokawa, D.; Murata, S.; Kitagawa, E.; Iwahashi, Y.; Nakagawa, R.; Hashido, T.; Iwahashi, H. Mechanisms of copper toxicity in Saccharomyces cerevisiae determined by microarray analysis. Environ. Toxicol. 2008, 23, 599-606. [CrossRef] [PubMed]

31. Liang, H.; Hu, Z.; Zhang, T.; Gong, T.; Chen, J.; Zhu, P.; Li, Y.; Yang, J. Production of a bioactive unnatural ginsenoside by metabolically engineered yeasts based on a new UDP-glycosyltransferase from Bacillus subtilis. Metab. Eng. 2017, 44, 60-69. [CrossRef] [PubMed]

32. Wang, P.; Wei, W.; Ye, W.; Li, X.; Zhao, W.; Yang, C.; Li, C.; Yan, X.; Zhou, Z. Synthesizing ginsenoside Rh2 in Saccharomyces cerevisiae cell factory at high-efficiency. Cell Discov. 2019, 5, 5. [CrossRef]

33. Wang, P.; Wei, Y.; Fan, Y.; Liu, Q.; Wei, W.; Yang, C.; Zhang, L.; Zhao, G.; Yue, J.; Yan, X.; et al. Production of bioactive ginsenosides Rh2 and Rg3 by metabolically engineered yeasts. Metab. Eng. 2015, 29, 97-105. [CrossRef]

34. Foresti, O.; Ruggiano, A.; Hannibal-Bach, H.K.; Ejsing, C.S.; Carvalho, P. Sterol homeostasis requires regulated degradation of squalene monooxygenase by the ubiquitin ligase Doa10/Teb4. Elife 2013, 2, e00953. [CrossRef] [PubMed]

35. Hu, Z.-F.; Gu, A.-D.; Liang, L.; Li, Y.; Gong, T.; Chen, J.-J.; Chen, T.-J.; Yang, J.L.; Ping Zhu, P. Construction and optimization of microbial cell factories for sustainable production of bioactive dammarenediol-II glucosides. Green Chem. 2019, 21, 3286-3299. [CrossRef]

36. Jensen, E.D.; Ferreira, R.; Jakociunas, T.; Arsovska, D.; Zhang, J.; Ding, L.; Smith, J.D.; David, F.; Nielsen, J.; Jensen, M.K.; et al. Transcriptional reprogramming in yeast using dCas9 and combinatorial gRNA strategies. Microb. Cell Fact. 2017, 16, 46. [CrossRef]

37. Smith, J.; Suresh, S.; Schlecht, U.; Wu, M.; Wagih, O.; Peltz, G.; Davis, R.W.; Steinmetz, L.M.; Parts, L.; Onge, R.P.S. Quantitative CRISPR interference screens in yeast identify chemical-genetic interactions and new rules for guide RNA design. Genome Biol. 2016, 17, 45. [CrossRef] [PubMed]

38. Vanegas, K.G.; Lehka, B.J.; Mortensen, U.H. SWITCH: A dynamic CRISPR tool for genome engineering and metabolic pathway control for cell factory construction in Saccharomyces cerevisiae. Microb. Cell Fact. 2017, 16, 25. [CrossRef]

39. Zhang, J.L.; Peng, Y.Z.; Liu, D.; Liu, H.; Cao, Y.X.; Li, B.Z.; Li, C.; Yuan, Y.J. Gene repression via multiplex gRNA strategy in Y. lipolytica. Microb. Cell Fact. 2018, 17, 62. [CrossRef]

40. Polakowski, T.; Stahl, U.; Lang, C. Overexpression of a cytosolic hydroxymethylglutaryl-CoA reductase leads to squalene accumulation in yeast. Appl. Microbiol. Biotechnol. 1998, 49, 66-71. [CrossRef] [PubMed] 
41. Thoma, R.; Schulz-Gasch, T.; D'Arcy, B.; Benz, J.; Aebi, J.D.; Dehmlow, H.; Hennig, M.; Stihle, M.; Ruf, A. Insight into steroid scaffold formation from the structure of human oxidosqualene cyclase. Nature 2004, 432, 118-122. [CrossRef] [PubMed]

42. Liang, Y.-L.; Zhao, S.-J.; Xu, L.-X.; Zhang, X.-Y. Heterologous expression of dammarenediol synthase gene in an engineered Saccharomyces cerevisiae. Lett. Appl. Microbiol. 2012, 55, 323-329. [CrossRef] [PubMed] 